05

\title{
Вариационный подход к определению динамической прочности материала
}

\author{
() А.Д. Евстифеев, ${ }^{1,2}$ Г.А. Волков ${ }^{2}$ \\ ${ }^{1}$ Научно-исследовательский институт механики Национального исследовательского Нижегородского государственного \\ университета им. Н.И. Лобачевского, \\ 603600 Нижний Новгород, Россия \\ ${ }^{2}$ Санкт-Петербургский государственный университет, \\ 199034 Санкт-Петербург, Россия \\ e-mail: ad.evstifeev@gmail.com
}

Поступило в Редакцию 7 сентября 2021 г.

В окончательной редакции 7 сентября 2021 г.

Принято к публикации 8 ноября 2021 г.

Рассмотрена проблематика определения механических характеристик малых образцов по методике Кольского с использованием разрезных стержней Гопкинсона. Представлены экспериментальные и теоретические данные по определению прочностных характеристик модельного материала при растяжении в квазистатическом и динамическом режимах. Показано, что возможные ошибки каждого отдельного эксперимента могут быть учтены в рамках структурно-временной модели разрушения в сочетании с алгоритмом обработки данных методом знаковозмущенных сумм.

Ключевые слова: динамическая прочность, критерий инкубационного времени, методика Кольского, метод знакопеременных сумм.

DOI: 10.21883/JTF.2022.02.52017.250-21

\section{Введение}

Оценка прочностных свойств материала при динамическом нагружении является общей практической задачей. Экспериментальные методы требуют тщательного и хорошо организованного процесса планирования каждого этапа испытания от подготовки образцов до обработки и интерпретации полученных данных с тем, чтобы оценить все факторы, влияющие на точность и повторяемость результатов. Отклонение измеряемого параметра от истинного значения может иметь как постоянную систематическую ошибку, так и случайную составляющую. Целью исследования является разработка методик, позволяющих учесть и исключить при оценке прочностных характеристик материала влияние случайных помех различной природы.

Одной из особенностей динамического тестирования является высокая вариативность результатов в зависимости от скорости нагружающего воздействия [17], а также прочих факторов, влияющих на характеристики материала. Следовательно, общепринятые вероятностные методы для случая гауссовского распределения случайных шумов не всегда способны обеспечить достоверный результат, а малое количество экспериментальных данных в широком диапазоне изменения параметров внешнего воздействия не позволяет применять статистический анализ, основанный на центральной предельной теореме. Таким образом, все вышеперечисленные проблемы приводят к отсутствию стандартного унифицированного инженерного подхо- да к оценке динамических прочностных свойств материалов.

Разрабатываемый метод экспериментально-аналитического анализа прочностных свойств материала основан на структурно-временном подходе к прогнозированию критического состояния разрушения при произвольной динамической нагрузке [8,9]. Преимущество этого подхода заключается в том, что свойства материала определяются не в рамках одного эксперимента, а по массиву экспериментальных точек, полученных при различных скоростных режимах. Предыдущие исследования показали высокую применимость структурновременного подхода к различным задачам механики и физики [10]. Значения модельных параметров в рамках настоящего исследования определяются неявно в соответствии с методом знакопеременных сумм (SPS - SignPerturbed Sums) [11]. Оценкой модельных параметров в рамках этого рандомизированного подхода является некоторый доверительный интервал, который содержит истинное значение параметра с заданной вероятностью, что в полной мере соответствует природе экспериментальных данных.

В качестве материала исследований был выбран медный сплав М1. Эксперименты выполнялись на динамической установке по растяжению с использованием разрезных стержней Гопкинсона. Расширение экспериментальных методик по определению динамических свойств материалов путем внедрения вариационных методов анализа позволило применить новую методологию тестирования материалов в рамках структурно-временного подхода. 


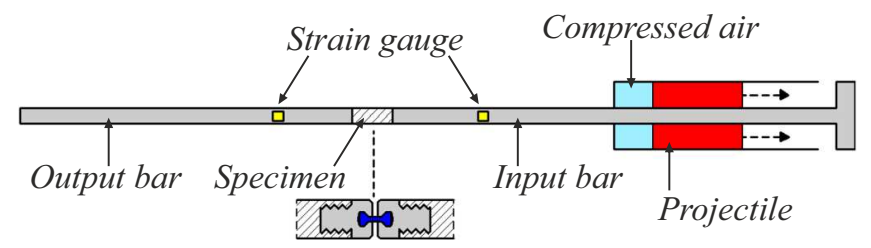

Рис. 1. Экспериментальная схема на растяжение, реализующая метод Кольского с использованием разрезных стержней Гопкинсона.

\section{1. Методика испытаний}

Эксперименты на растяжение в области квазистатического режима нагружения выполнялись на разрывной машине Shimadzu AG-50kNX. Эксперименты на динамическое растяжение выполнялись на установке, реализующей метод Кольского с использованием разрезных стержней Гопкинсона $[12,13]$. Схема установки представлена на рис. 1. Установка состоит из пневматически нагружающего устройства калибром $27 \mathrm{~mm}$, ударника длиной $400 \mathrm{~mm}$, разрезных стержней Гопкинсона диаметром $16 \mathrm{~mm}$ и длиной нагружающего стержня $3000 \mathrm{~mm}$, а также мерного стержня $-1500 \mathrm{~mm}$. Для разгона ударника сжатый воздух нагнетается компрессором в камеру, давление контролируется манометром. Время открытия клапана и синхронизация сигналов осуществляется программным комплексом на базе микрокомпьютера Arduino. После открытия клапана ударник разгоняется и бьет по наковальне, жестко связанной с нагружающим стержнем. В нагружающем стержне формируется импульс растяжения, регистрируемый тензорезисторами с использованием высокочастотного усилителя. Прошедший через образец импульс регистрируется аналогичным образом на опорном стержне.

Образцы из меди М1 изготавливались в виде лопаток с геометрическими размерами рабочей части $5 \mathrm{~mm}$ в длину и $2 \mathrm{~mm}$ в ширину. С целью снижения погрешности при смене экспериментальных схем были изготовлены идентичные фиксирующие захваты для динамической установки и статической разрывной машины. Экспериментальная схема по динамическому растяжению малых образцов и правомерность ее использования обсуждалась в [14] при сравнении данных испытаний образцов стандарта ISO-8256 и малых образцов на башенном копре с ускорителем.

В качестве пороговых величин, характеризующих разрушение образца, в настоящей работе рассматривается зависимость максимального разрывающего напряжения от скорости роста напряжения. Стоит отметить, что при получении сигнала от растяжения малого образца низкой прочности использовался усилитель с коэффициентом усиления $100 \times$, что накладывает существенные ограничения на частотный диапазон его работы. При частотах до $200 \mathrm{kHz}$ происходит усиление сигнала без искажения оригинала. С ростом частоты достоверность фиксируемого сигнала снижается.

Другим аспектом при работе с численным массивом данных является необходимость минимизации случайной ошибки, связанной с ручным (экспертным) анализом данных. В рамках настоящей работы целевым значением по каждому единичному эксперименту является пара, состоящая из прочности на растяжение и скорости роста напряжения. Величина прочности материала зависит от способа фильтрации и сглаживания исходного сигнала, и при этом никаких вопросов при его определении не возникает. Обратная ситуация возникает при оценке скорости роста напряжений, поэтому с целью минимизации ошибки ее определения предлагается использовать автоматизированный алгоритм в каждом эксперименте. Рассмотрим на примере диаграммы напряжений при растяжении образца из медного сплава М1 при скорости роста напряжения $19000 \mathrm{GPa} / \mathrm{s}$ (рис. 2) работу предложенного алгоритма. Профиль сигнала, соответствующий первому проходу волны растяжения через образец и мерный стержень, выделяется из записанного осциллографом массива данных автоматически. При экспертном анализе графика за временное сопротивление разрыву может быть принята величина $\sigma_{U T S}=\sigma_{2}$, а за скорость нагружения $\dot{\sigma}=\left(\sigma_{2}-\sigma_{1}\right) /\left(t_{2}-t_{1}\right)$. Однако диаграммы растяжения во времени могут существенно отличаться от представленной на рис. 2 по причине случайных шумов, различия в контактном взаимодействии на начальном этапе растяжения образца и захватов, первичной оцифровки общего сигнала и т. д. В автоматизированной схеме использован следующий алгоритм:

1. В цикле по времени определяется время $\left(t_{2}\right)$, соответствующее максимальному значению напряжения $\left(\sigma_{U T S}=\sigma_{2}\right)$, а также общее время эксперимента $\left(t_{\text {full }}\right)$.

2. На основе полученного значения фиксируются три интервала величиной $d t_{1}=0.3 \cdot t_{2}, d t_{2}=0.4 \cdot d t_{2}$, $d t_{3}=0.5 \cdot t_{2}$.

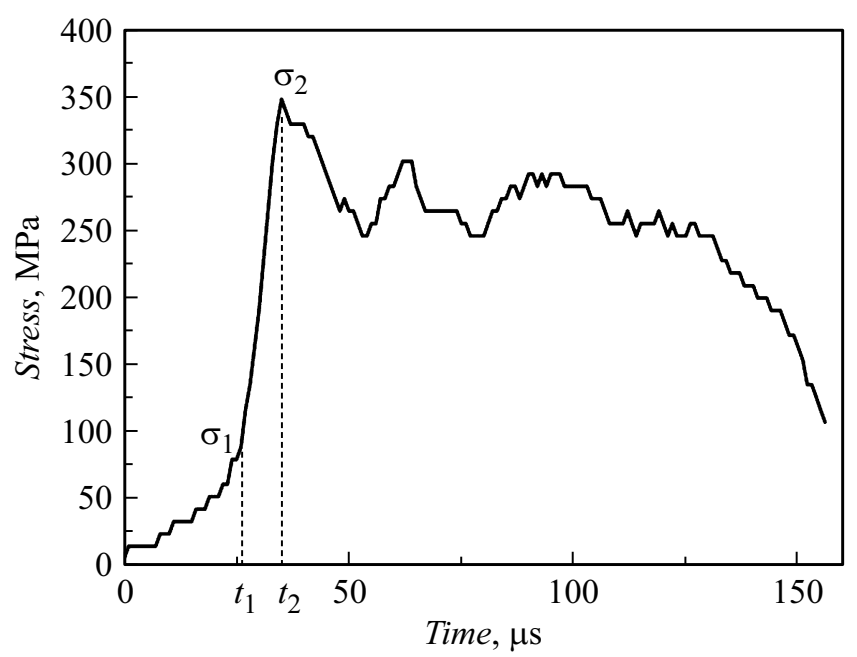

Рис. 2. Диаграмма напряжений при растяжении образца из медного сплава М1 при скорости роста напряжения $19000 \mathrm{GPa} / \mathrm{s}$. 


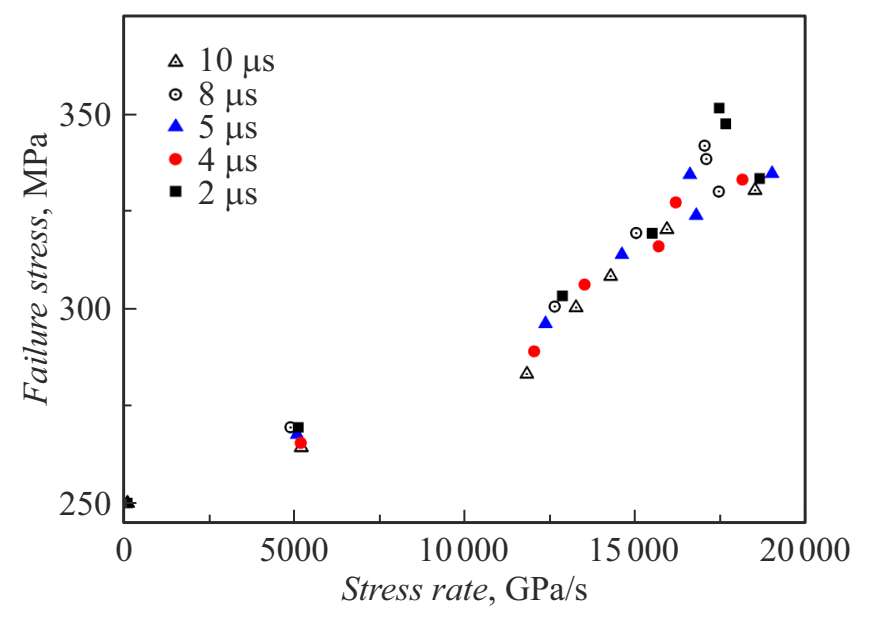

Рис. 3. Зависимость максимальной прочности на растяжения меди М1 от скорости роста напряжения при вариации параметров осреднении исходного импульса.

3. В цикле для каждого из значений интервала определяется $\dot{\sigma}_{j}=\max _{i}\left(\sigma\left(t_{i}+d t_{j}\right)-\sigma\left(t_{i}\right)\right) / d t_{j}$, где $j=1 \ldots 3$, a $t_{i}=0 \ldots\left(t_{\text {full }}-d t_{j}\right)$.

4. В качестве скорости роста напряжения для каждого эксперимента выбирается среднее значение $\dot{\sigma}=\left(\dot{\sigma}_{1}+\dot{\sigma}_{2}+\dot{\sigma}_{3}\right) / 3$

Предложенный подход был апробирован на большом количестве экспериментальных данных с различным профилем растягивающего напряжения и позволяет в автоматизированном режиме определять скорость роста напряжения с высокой стабильностью.

В качестве первичной обработки массивов экспериментальных данных, предшествующей вышеописанному алгоритму, можно рассмотреть процедуру скользящего среднего для полученного профиля сигнала с вариацией ширины отрезка осреднения. Были рассмотрены отрезки шириной $2,4,5,8$ и $10 \mu \mathrm{s}$, что соответствует частотам дискретизации $500,250,200,125$ и $100 \mathrm{kHz}$ соответственно. Зависимость максимальной прочности на растяжение меди M1 от скорости роста напряжения, определенная, согласно предложенному алгоритму, при вариации параметров осреднения исходного импульса представлена на рис. 3. Каждому испытанию соответствует пять точек на графике, что создает дополнительный массив данных в условиях точечных экспериментов. При этом стоит отметить, что процедура скользящего среднего при вариации ширины отрезка осреднения по времени корректирует положение точки на графике как по оси абсцисс, так и по оси ординат.

Анализируя экспериментальные данные, представленные на рис. 3, можно отметить зависимость максимальной прочности на растяжение медных образцов от скорости роста напряжения. С ростом скорости происходит увеличение пиковых значений от $250 \mathrm{MPa}$ при квазистатическом нагружении до $350 \mathrm{MPa}$ при скорости роста напряжения порядка $20000 \mathrm{GPa} / \mathrm{s}$. Стоит отметить, что с увеличением скорости роста напряжения увеличивается разброс при определении экстремальных значений напряжений.

\section{2. Анализ экспериментальных данных}

Для анализа прочностных характеристик материала в широком диапазоне изменения параметров нагружающего импульса далее в качестве критерия разрушения материала при растяжении возьмем хорошо зарекомендовавший себя критерий инкубационного времени [10]:

$$
\frac{1}{\tau} \int_{t-\tau}^{t} \frac{\sigma(s)}{\sigma_{U T S}} d s \leq 1,
$$

где $t-$ время, $\sigma$ - зависимость разрывающего напряжения от времени, $\sigma_{U T S}-$ временное сопротивление разрыву при квазистатическом нагружении, $\tau$ инкубационное время разрушения, отвечающее за динамическую прочность материала. В предположении линейного роста напряжений с постоянной скоростью $\dot{\sigma}$ в образце до момента разрушения или начала процессов необратимого деформирования скоростная зависимость критического уровня напряжений может быть вычислена аналитически по следующей формуле:

$$
\sigma_{*}(\dot{\sigma})=\varphi(\tau, \dot{\sigma})= \begin{cases}\sigma_{U T S}+\frac{\tau}{2} \dot{\sigma}, & \dot{\sigma} \leq \frac{2 \sigma_{U T S}}{\tau}, \\ \sqrt{2 \sigma_{U T S} \tau \dot{\sigma},} & \dot{\sigma}>\frac{2 \sigma_{U T S}}{\tau}\end{cases}
$$

С применением метода наименьших квадратов (МНК) были вычислены оптимальные значения параметра $\tau$, минимизирующие среднеквадратичное отклонение расчетной зависимости (2) от экспериментальных точек. Для каждой группы значений частоты дискретизации были определены значения инкубационного времени и построены диаграммы максимального разрывающего напряжения от скорости роста напряжения. На рис. 4 представлены расчетные и экспериментальные зависимости максимальной прочности на растяжение от скорости роста напряжения для частоты дискретизации временного профиля разрывающего напряжения 500 и $100 \mathrm{kHz}$. Расчетные кривые построены по критерию (1) с учетом параметров $\sigma_{U T S}=250 \mathrm{MPa}$ и $\tau_{500 \mathrm{kHz}}=9.9 \mu \mathrm{s}, \tau_{100 \mathrm{kHz}}=7.6 \mu \mathrm{s}$. Для других вариаций временного отрезка осреднения параметры $\tau_{250 \mathrm{kHz}}=9.5 \mu \mathrm{s}, \tau_{200 \mathrm{kHz}}=8.9 \mu \mathrm{s}$ и $\tau_{125 \mathrm{kHz}}=8.3 \mu \mathrm{s}$ соответственно. Среднее значение параметра для всего массива точек $\tau=8.8 \mu \mathrm{s}$. Метод МНК обеспечивает точечную оценку, но не позволяет определить степень достоверности интервала вычисляемого параметра, особенно в условиях вариативности исходных данных. Стоит отметить, что среднее значение параметра инкубационного времени оказалось близко к величине, определенной для массива данных, сформированного 
для частоты дискретизации временного профиля разрывающего напряжения $200 \mathrm{kHz}$, что соответствует частотному порогу усилителя сигнала при усилении $100 \times$. При увеличении частоты исходный сигнал искажается при прохождении через усилитель, а при уменьшении частоты искажение сигнала происходит численно из-за чрезмерного усреднения.

Значения инкубационного времени, вычисленные для различных частот дискретизации, принадлежат интервалу $[7.6 ; 9.9] \mu \mathrm{s}$. С целью дальнейшего исследования было проведено сравнение полученного диапазона значений с доверительным интервалом, определяемым методом знаковозмущенных сумм. Ранее этот метод хорошо себя зарекомендовал при решении задач оценки параметров системы в условиях неопределенности шума и недостатка данных. Было показано, что SPS-метод может быть обоснованно применен в рамках структурно-временного подхода для оценки значений инкубационного времени $[14,15]$. Для расчета были выбраны экспериментальные данные, полученные для частоты дискретизации временного профиля $200 \mathrm{kHz}$. Для уровня достоверности
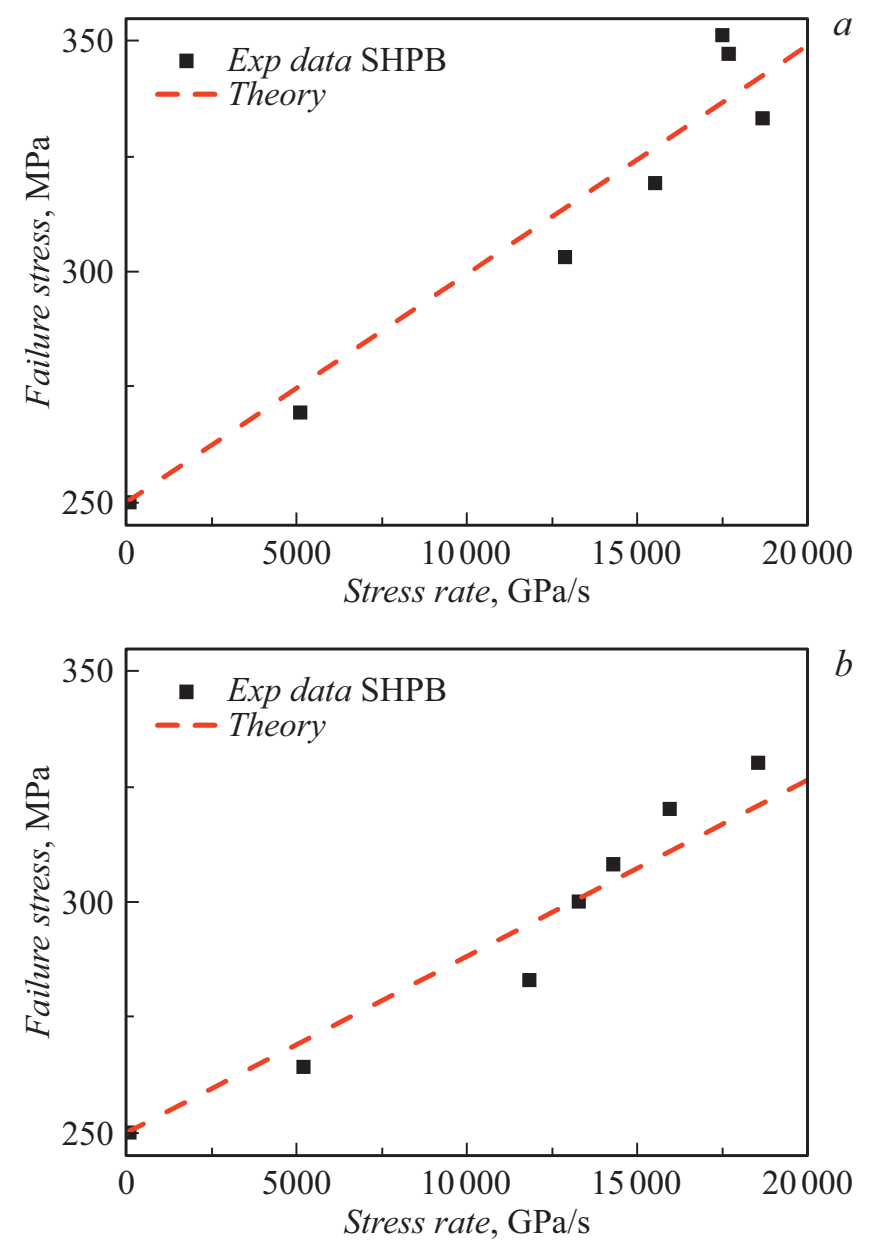

Рис. 4. Расчетная и экспериментальная зависимости максимальной прочности на растяжение от скорости роста напряжения для частоты дискретизации временного профиля разрывающего напряжения $a-500$ и $b-100 \mathrm{kHz}$.

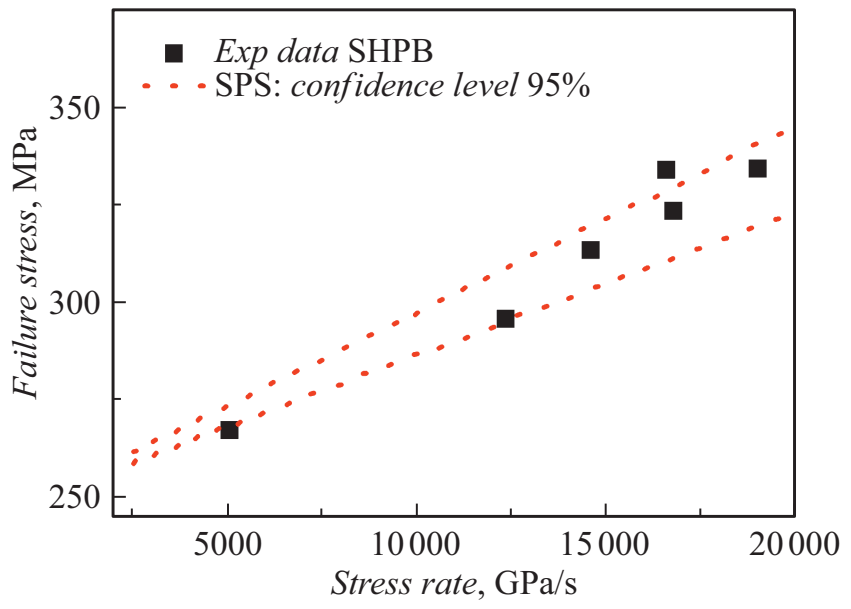

Рис. 5. Вычисленная методом знаковозмущенных сумм зависимость максимальной прочности на растяжение от скорости роста напряжения для частоты дискретизации временного профиля разрывающего напряжения $200 \mathrm{kHz}$ в сравнении с экспериментальными данными.

95\% была вычислена оценка для инкубационного времени в виде интервала $\tau \in[7.33 ; 9.53] \mu \mathrm{s}$ (рис. 5), который практически совпадает с полученным ранее диапазоном значений для различных частот дискретизации. Это позволяет сделать вывод о том, что оценка значения инкубационного времени методом знаковозмущенных сумм позволяет избежать существенных отклонений вычисляемой величины параметра инкубационного времени от своего средневзвешенного значения даже в условиях небольшого количества экспериментальных точек. В приведенном примере было показано, что доверительный интервал, определяемый методом знаковозмущенных сумм, соответствует интервалу, полученному путем введения в характеристики сигнала дополнительного отклонения, соответствующего как избыточному осреднению, так и реальным помехам, формируемым усилителем на высоких частотах. Модельный эксперимент позволил искусственно сформировать расширенный набор „экспериментальных“ точек и верифицировать результаты расчета в рамках структурно временного подхода путем определения доверительного интервала.

\section{Заключение}

В работе на примере медного сплава M1 были исследованы прочностные характеристики материала в квазистатическом и динамическом режимах нагружения. Экспериментально были получены хронограммы разрывающего напряжения на установке, реализующей методику Кольского с использованием разрезных стрежней Гопкинсона, при различных скоростях ударника. Показано, что исходные диаграммы не позволяют однозначно определить прочностные характеристики материала. 
Была разработана методика анализа данных испытаний, включающая автоматизированный алгоритм оцифровки массива экспериментальных данных и оценки скоростной зависимости прочности. К прогнозированию критического состояния разрушения при произвольной динамической нагрузке был применен структурновременной подход с использованием SPS-алгоритма.

\section{Финансирование работы}

Исследование выполнено при финансовой поддержке РФФИ (№ 19-31-60031, № 20-31-70053).

\section{Конфликт интересов}

Авторы заявляют, что у них нет конфликта интересов.

\section{Список литературы}

[1] G.V. Stepanov, V.V. Astanin, V.I. Romanchenko, A.P. Vashchenko, V.M. Tokarev, B.D. Chukhin, Y.P. Guk. Strength of Materials, 15, 220 (1983).

[2] А.М. Брагов, А.К. Ломунов. ПМТФ, 5, 168 (1988).

[3] A.M. Bragov, B.L. Karihaloo, Yu.V. Petrov, A.Yu. Konstantinov, D.A. Lamzin, A.K. Lomunov, I.V. Smirnov. J. Appl. Mechan. Tech. Phys., 53 (6), 926 (2012).

[4] A.A. Gruzdkov, E.V. Sitnikova, N.F. Morozov, Y.V. Petrov. Mathem. Mechan. Solids, 14, 72 (2009).

[5] А.А. Груздков, С.И. Кривошеев, Ю.В. Петров. ФТТ, 45 (5), 842 (2003). [A.A. Gruzdkov, S.I. Krivosheev, Y.V. Petrov. Physics Solid State, 45, 886 (2003).]

[6] G.I. Kanel, S.V. Razorenov, A.A. Bogatch, A.V. Utkin, V.E. Fortov, D.E. Grady. J. Appl. Phys., 20, 467 (1997).

[7] Г.В. Гаркушин, Г.И. Канель, С.В. Разоренов. ФТТ, 52, 2216 (2010).

[8] Y.V. Petrov, A.A. Utkin. Sov. Mater. Sci., 25, 153 (1989).

[9] Y.V. Petrov, N.F. Morozov. ASME J. Appl. Mech., 61, 710 (1994).

[10] Yu.V. Petrov. Dokl. Akademii Nauk, 395 (5), 621 (2004).

[11] B.C. Csaji, M.C. Campi, E. Weyer. IEEE Transactions on Signal Processing, 63 (1), 169 (2015).

[12] H. Kolsky, Proceedings of the Physical Society, 62, 676 (1949).

[13] A.M. Bragov, A.K. Lomunov. Int. J. Impact. Engin., 16 (2), 321 (1995).

[14] А.Д. Евстифеев, Г.А. Волков, А.А. Чеврычкина, Ю.В. Петров. ЖТФ, 89 (4), 567 (2019). [A.D. Evstifeev, G.A. Volkov, A.A. Chevrychkina, Y.V. Petrov. Tech. Phys., 64 (4), 523 (2019).]

[15] M.V. Volkova, O.N. Granichin, G.A. Volkov, Y.V. Petrov. Vestnik St. Petersburg University: Mathematics, 51 (1), 23 (2018). 